\title{
Fanconi anemia: from DNA repair to metabolism
}

\author{
Silvia Ravera $\mathbb{D}^{1} \cdot$ Carlo Dufour $^{2} \cdot$ Paolo Degan $^{3} \cdot$ Enrico Cappelli $^{2}$
}

Received: 21 September 2017 / Revised: 21 September 2017 / Accepted: 27 October 2017 / Published online: 2 February 2018

(c) European Society of Human Genetics 2018

Fanconi anemia (FA) is a genetic disease caused by mutations in at least 21 genes. Even if the clinical phenotype of FA patients is heterogeneous, the main clinical aspects are somatic malformations, bone marrow failure and predisposition to develop cancer, in particular acute myeloid leukemia (AML) and epithelial cancer of head and neck [1]. Regarding the molecular causes of the disease, the impairment of DNA repair has been considered the principal defect in FA cells. However, in the last few years, other contributors to DNA repair defects have emerged. They include mitochondrial function alterations and the consequent reduction of energy production and the increment of oxidative stress [2], the defects in mitophagy, the reduced ability of aldehyde detoxification, the hyperproduction and the hyper sensitivity to inflammatory cytokines [3]. This view seems to be strengthened by the recent publication in this journal about the case of a female child with a clinical and cellular phenotype largely compatible with a diagnosis of FA, consisting in failure to thrive, minor dysmorphic features and a positive chromosomal fragility test ( $29 \%$ of the cells showing $\geq 1$ break/cell after exposure to DEB) [4], which is still considered the diagnostic gold standard for FA [1]. This patient did not carry mutations in any of the currently known FA genes, but displayed a mutation only in $\operatorname{COX} 4-1$, a gene codifying for a mitochondrial protein involved in the oxidative phosphorylation [4].

The case of this patient suggests shifting the focus from defect of DNA repair to the energy metabolism alteration in

Enrico Cappelli

enricocappelli@gaslini.org

1 Department of Pharmacy, Biochemistry Laboratory, University of Genova, Genova, Italy

2 Hematology Unit Istituto Giannina Gaslini, Genova, Italy

3 U.O. Mutagenesi e Prevenzione Oncologica IRCCS AOU San Martino-IST (Istituto Nazionale per la Ricerca sul Cancro), Genova, Italy
FA. Indeed, FA cells display defects in respiratory chain, generating a decrement of energetic status and an enhancement of the oxidative stress [2], a biochemical framework very close to the one described in Abu-Libdeh paper.

This suggests that even if DNA repair defects have a clear role in determining important clinical aspects of FA patients such as the neoplastic transformation and the hematopoietic stem cells impoverishment, the functional defects associated with the altered mitochondrial physiology also provide an important contribution to FA symptoms. Apparently, these defects converge into a prooxidation phenotype associated with FA. Indeed an improvement of the chromosomal stability in lymphocytes from FA patients [5] and a reduction of the cytotoxic effects of TNF-alpha [6] have been observed after treatment of FA cells with selected antioxidants. Moreover, other antioxidants have been shown to improve insulin resistance and obesity in FA mice [7] and to restore the metallo-proteinases activity in FA cells thus suggesting a possible role of oxidative stress in the glucose and lipid metabolic disturbance and in somatic malformations [8].

Overall, this evidence may lead to looking at DNA repair defect as an epiphenomenon of other perturbed functions: the high DNA damage of FA cells may be correlated with the oxidative stress caused by altered mitochondrial energy metabolism, as well as the reduction of aldehyde detoxification. This hypothesis is in line with the heterogeneity of the disease that better fits with an articulated and complex metabolic defect rather than with a mere DNArepair disease. Under this perspective it is worth noting that 30 years ago, Joenje et al. [9] showed how chromosomal aberrations were oxygen-dependent in Fanconi anaemia lymphocytes.

So far, mainstream research has tried to associate FA pathology only to the DNA repair defect perspective, neglecting other factors that might turn out to be the root causes. Indeed, evidence provided in Abu-Libdeh's work 
seems to strongly invite us to consider this as a realistic approach to the disease.

The above reported remarks pave the way for a serious discussion upon possible antioxidant therapy, a question that might not be limited to the Fanconi's anemia context but could rather be extended to a wide range of pathologies that may well benefit from this type of approach.

Remaining within FA it would be necessary to establish which antioxidants may be appropriate in the context of a pathology that demonstrates alterations in the mitochondrial functions and in energy metabolism.

In conclusion, we believe that the evaluation of the energy metabolism alterations could open new horizons for FA patients diagnosis and therapy. This can also hold true for other bone marrow failure diseases, which share with FA some common features [10].

For reply see Brief Communication/Short Report by Douiev et al. in this issue.

Acknowledgements We want to acknowledge AIRFA for supporting the activity of the Clinical and Experimental Hematology Unit of the G. Gaslini Institute.

\section{Compliance with ethical standards}

Conflict of interest No Authors have conflict of interest.

\section{References}

1. Dufour C. How I manage patients with Fanconi anaemia. $\mathrm{Br} \mathbf{J}$ Haematol. 2017;178:32-47.

2. Cappelli E, Ravera S, Vaccaro D, et al. Mitochondrial respiratory complex I defects in Fanconi anemia. Trends Mol Med. 2013;19:513-4.

3. Cheung RS, Taniguchi T. Recent insights into the molecular basis of Fanconi anemia: genes, modifiers, and drivers. Int J Hematol. 2017;106:335-44.

4. Abu-Libdeh B, Douiev L, Amro S, et al. Mutation in the COX4I1 gene is associated with short stature, poor weight gain and increased chromosomal breaks, simulating Fanconi anemia. Eur J Hum Genet. 2017. https://doi.org/10.1038/ejhg.2017.112.

5. Ponte F, Sousa R, Fernandes AP, et al. Improvement of genetic stability in lymphocytes from Fanconi anemia patients through the combined effect of $\alpha$-lipoic acid and $\mathrm{N}$-acetylcysteine. Orphanet $\mathrm{J}$ Rare Dis. 2012;7:28.

6. Zhang X, Sejas DP, Qiu Y, Williams DA, Pang Q. Inflammatory ROS promote and cooperate with the Fanconi anemia mutation for hematopoietic senescence. J Cell Sci. 2007;120:1572-83.

7. Li J, Sipple J, Maynard S, et al. Fanconi anemia links reactive oxygen species to insulin resistance and obesity. Antioxid Redox Signal. 2012;17:1083-98.

8. Ravera S, Capanni C, Tognotti D, et al. Inhibition of metalloproteinase activity in FANCA Is linked to altered oxygen metabolism. J Cell Physiol. 2015;230. https://doi.org/10.1002/jcp. 24778.

9. Joenje H, Arwert F, Eriksson AW, de Koning H, Oostra AB. Oxygen-dependence of chromosomal aberrations in Fanconi's anaemia. Nature. 1981;290:142-3.

10. Degan P, Ravera S, Cappelli E. Why is an energy metabolic defect the common outcome in BMFS? Cell Cycle. 2016;15. https://doi. org/10.1080/15384101.2016.1218103. 